\title{
Comunicación corporativa para la fidelización del cliente
}

\section{Corporate communication for customer loyalty}

\section{Comunicação corporativa para a fidelidade do cliente}

\section{PATRICIA DURÁNBRAVO}

patricia.duran@correo.buap.mx - Benemérita Universidad Autónoma de Puebla, México.

ORCID: https://orcid.org/0000-0003-2388-2432

\section{NANCY CISNEROS MARTÍNEZ}

nancy.cisneros@correo.buap.mx - Benemérita Universidad Autónoma de Puebla, México.

ORCID: https://orcid.org/ 0000-0003-4636-5374

\section{RAÚL PANCARDO PERALTA}

rpancardo@gmail.com - Benemérita Universidad Autónoma de Puebla, México.

ORCID: https://orcid.org/0000-0002-0763-3762

Fecha de recepción: 31 de enero de 2020

Fecha de aceptación: 10 de mayo de 2020

\section{RESUMEN}

La propuesta del modelo estratégico para diagnosticarla comunicación corporativa orientadaa mejorarlafidelización del cliente fue desarrollada a partir de tres genealogías:la comprensión dela complejidad del contextoorganizacionaldeScheinsohn (2009), el BrandingCorporativo de Capriotti (2009) yel Proceso Comercial Relacional de Chiesa (2005). Con base en estas propuestas teóricas, se propone un Modelo estratégico de diagnóstico de la comunicación corporativa, con un paquete metodológico que permita identificar la carga informativa, relacional y simbólica, para orientar estratégicamente los activos intangibles como la identidad, la imagen y la reputación de la organización hacia los programas de atracción, satisfacción y fidelización del cliente.

PALABRAS CLAVE: comunicación corporativa, fidelización del cliente, diagnóstico organizacional.

\section{ABSTRACT}

The proposal of the strategic model to diagnose corporate communication aimed at improving customer loyalty developed from threegenealogies: the understanding of 
the complexity of the organizational context of Scheinsohn (2009), the Corporate Branding of Capriotti (2009) and the Process Chiesa Relational Commercial (2005). Based on these theoretical proposals, it proposes a Strategic diagnostic model for corporate communication, with a methodology package that identifies the informational, relational and symbolic burden, to strategically guide intangible assets such as the identity, image and reputation of the organization, towards customer attraction, satisfaction and loyalty programs.

KEYWORDS: corporate communication, customer loyalty, organizational diagnosis.

\section{RESUMO}

A proposta do modelo estratégico para diagnosticar a comunicação corporativa com o objetivo de melhorar a fidelidade do cliente foi desenvolvida a partir de três genealogias: a compreensão da complexidade do contexto organizacional de Scheinsohn (2009), a Marca Corporativa de Capriotti (2009) e o Processo Comercial relacional da Chiesa (2005). Com base nessas propostas teóricas, propõe um modelo de diagnóstico estratégico para a comunicação corporativa, com um pacote de metodologia que identifica a carga informacional, relacional e simbólica, para orientar estrategicamente ativos intangíveis, como identidade, imagem e reputação da organização, programas de atração, satisfação e fidelização de clientes.

PALAVRAS-CHAVE: comunicação corporativa, fidelização do cliente, diagnóstico organizacional. 


\section{INTRODUCCIÓN}

La fidelización del cliente (en adelante, FC) es el proceso que busca construir relaciones de largo plazo que agreguen valor para conservar a los clientes más rentables (Castelló-Martínez, 2010). En este proceso la comunicación corporativa (en adelante, CC) juega un papel fundamental, pues se convierte en el sistema generador de esas interacciones y relaciones sustentables entre la empresa y sus clientes.

El Barómetro de Fidelización de la IE University señala que es más rentable fidelizar a los clientes que conseguir nuevos: 8 de cada 10 empresas consideran que es más costoso atraer nuevos clientes que retener a los cautivos (Marketing, 2018). Para las pequeñas y medianas empresas (Pymes) el reto es mayor, debido al costo que implica la captación de nuevos clientes: el gasto de captar nuevos clientes llega a ser hasta siete veces mayor que fidelizarlos (Dir\&Age, 2018).

La comunicación corporativa permite el "direccionamiento estratégico de las herramientas de comunicación que contribuyen a la notoriedad y a la construcción de vínculos hacia los stakeholders, aportando al logro de los objetivos planteados" (Apolo, Baez, Pauker \& Pasquel, 2017, p. 527). De ahíla importancia de proponer un modelo estratégico que permita hacer un diagnóstico organizacional de la comunicación corporativa teniendo en cuenta la identidad, la imagen y la reputación de la organización, para generar relaciones de valor que se traduzcan en la FC.

\section{PLANTEAMIENTO DEL PROBLEMA}

El Modelo estratégico de diagnóstico de la CC orientado a mejorar la FC que aquí se presenta se desarrolla en el marco de la selección investigativa y un estudio de caso centrado en la tienda de autoservicios Sanver, que forma parte de una cadena de doce sucursales de un grupo empresarial mexicano orientado a la comercialización de las marcas líderes del ramo ferretero y de la construcción.

A la hora de definir su "Filosofía corporativa", la empresa declara estar "comprometida con sus clientes para satisfacer plenamente sus requerimientos yexpectativas buscandola excelencia de su servicio mediante el aseguramiento de la calidad y el trabajo en equipo" (Sanver,2018,p.5). Sin embargo, la empresa ha registrado resultados negativos en la autoevaluación anual de "Satisfacción al Cliente", como se muestra en el Gráfico 1: 


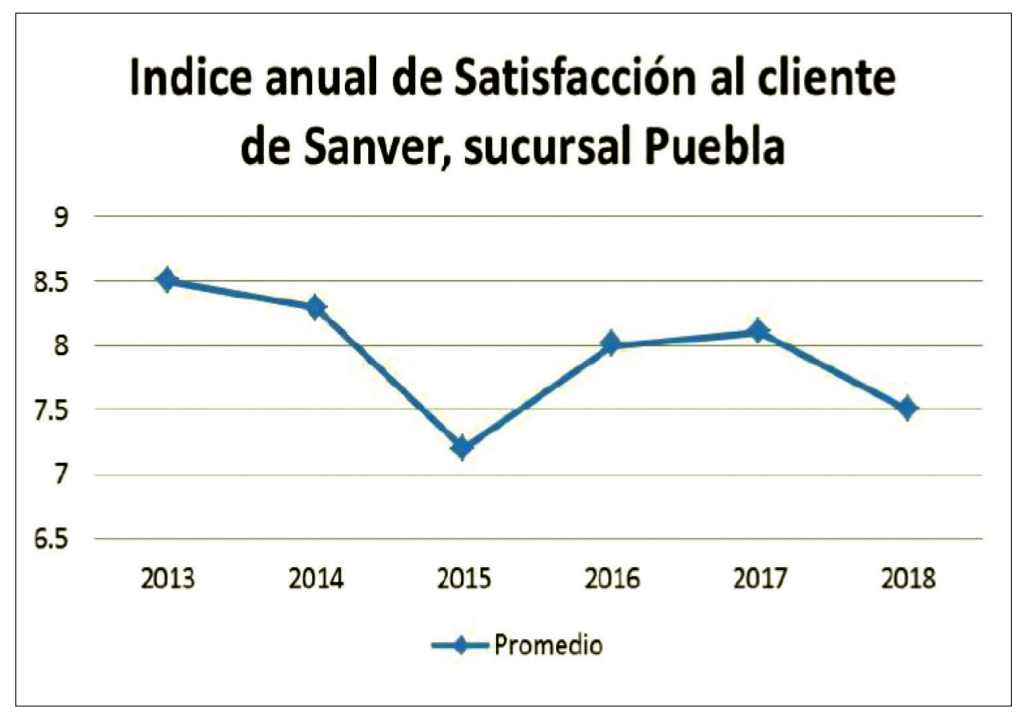

Fuente: Sanver, Sistema de Gestión de la calidad, Mercadotecnia, Reportes (Sanver 2018).

Pese a conocer estas cifras, la empresa no ha podido generar una estrategia corporativa eficaz que le ayude a revertir la tendencia adversa, no sólo para mejorar la satisfacción del consumidor, sino para trascender hacia la FC.

Para lograr el propósito de FC, es necesario no sólo evaluar la información transmitida en el momento de la compra, también es importante valorar las experiencias en relación al producto, los empleados y la organización en general. En este sentido, Capriotti (2009) explica quela organización, en el ejercicio dela CC, establece un diálogo social a través de "información socialmente mediada e información directamente experimentada" (p. 45).

Con base en lo anterior, se detectaron deficiencias en el proceso de evaluación que aplica la empresa, tanto en el método como en el instrumento de medición. Se puede observar una mirada reducida del proceso en el que se consideran únicamente dos actores: el cliente y el empleado; por otra parte, sólo se centra en el momento de la compra; y, además, se enfoca en la valoración de constructos como: la "atención del asesor de ventas", "los precios", "las promociones"; si el cliente "encontró todo lo que buscaba" y si "recomendaría comprar en la tienda". Por último, deja un apartado libre para que los clientes puedan incluir otras observaciones con respecto a su experiencia de compra en la tienda. 
Figura 1. Encuesta de satisfacción al cliente Sanver (Sanver, 2013)

Encuesta de satisfacción al cliente
Nombre:
Sus comentarios son la base de nuestras mejoras, ayúdenos a continuar este camino
para seguir brindandole el servicio que usted se merece. Le pedimos conteste honesta
y objetivamente las siguientes preguntas:

Fuente: Sanver, Documentos internos, Sistema de Gestión de la calidad, Mercadotecnia, PGM-05.

Con los resultados de esta evaluación se recaban datos sobrela información transmitida al cliente. No obstante, se ignora la amplitud y la complejidad de los elementos que directa o indirectamente contribuyen a lograr la FC. Por ello, surge el interés de desarrollar un modelo para diagnosticar la CC desde una perspectiva estratégica, sistémica y situacional, que pueda ser adaptable y replicable en los diversos puntos de ventas en donde la empresa opera en el estado de Puebla.

\subsection{Primer momento: el mapa relacional organizacional}

La actividad de FC es un proceso complejo que requiere identificar la diversidad de elementos y procesos involucrados. Primero, es necesario ampliar el mapa relacional a fin de reconocer el sistema de intercambios que surgen tanto dentro como fuera de la organización, dada su condición de sistema. En tal sentido, Garrido (2007) asevera que "la empresa se interrelaciona e interactúa constantemente con su medio debido a que es un sistema vivo y abierto estableciendo vínculos constantes con su entorno social” (p. 19).

Sobre este aspecto, Scheinsohn (2009) señala que la empresa es un sistema asociado a una red amplia de relaciones sociales, en el que identifica tres niveles: el sistema empresa, el suprasistema y el macrosistema. Todos y cada uno de estos componentes del sistema influyen de manera directa o indirecta en los procesos de la organización debido a su interdependencia.

El análisis del macrosistema se desarrolla con la revisión de factores externos (educacional, político, económico, legal, cultural, científico, tecnológico, demográfico, sociológico y recursos naturales, entre otros), con la finalidad de observar la interacción que la empresa mantiene con su entorno, proceso fundamental para el funcionamiento del sistema. Mientras que para el sistema 
empresa se propone un análisis basado en el modelo sociotécnico abierto de Kast y Rosenzweig (1990), en cuyo marco se plantea que el sistema de la empresa está constituido por cinco subsistemas principales: el subsistema filosófico, técnico, estructural, psicosocial y administrativo.

Finalmente, para establecer la densidad y el tipo de relaciones con los stakeholders que conforman el suprasistema, se utilizan los aportes de tres autores que han contribuido al diagnóstico de este tipo de vínculos entre las organizaciones y sus públicos. El "Análisis de públicos" de Capriotti (2009), la propuesta de las cuatro "Categorías de posibles contactos" de Chiesa, (2013) y el "Reconocimiento de actores" de Massoni (2013).

Con el "Análisis de públicos" de Capriotti (2009) es posible reconocer todos aquellos grupos con los que la organización tiene algún tipo de contacto, pero ademássepuedeidentificar el papel prioritario delosempleadosyclientes, sin descuidar la influencia que los públicos secundarios ejercen sobre el proceso de FC.

Posteriormente, es necesario considerar la propuesta de las cuatro "Categorías de posibles contactos” de Chiesa (2013), con el propósito de orientar este mapa relacional hacia el objetivo del estudio, la FC, y para determinar el tipo de relación de cada uno de los públicos con la organización, en base a la siguiente clasificación:

- Clientes potenciales: Son todos aquellos prospectos que, por su actividad, representan un segmento atractivo para la empresa, pero aún no tienen contacto con ella.

- Compradores: Son todas aquellas personas u organizaciones que sólo han realizado una compra o que sólo acuden a comparar precios.

- Clientes: Son las personas que registran dos o más experiencias de compra con la empresa. Es un cliente satisfecho.

- Socioamigo: Es el cliente que no sólo repite sus compras y concreta un amplio consumo; también es el que recomienda y mantiene un trato más cercano con la empresa. Es un cliente fiel.

Finalmente, se integra la propuesta de "Reconocimiento de actores" de Massoni (2013), con la que es posible profundizar el análisis de los empleados, en quienes se identifican tres roles posibles:

- Empleados que atienden: encargados de la atención directa al cliente en el punto de venta.

- Empleados que administran: dedicados a tareas administrativas de soporte para la atención al cliente, con quien no tienen contacto directo.

- Empleados que motivan: orientan, supervisan y capacitan a los clientes. 
El resultado del ejercicio de análisis relacional dela organización se muestra en la Figura 2, un mapa estratégico que permite identificar la multidimensionalidad y la complejidad de relaciones funcionales y simbólicas en la que se encuentra inmersa la organización.

Figura 2. Mapa relacional de la organización

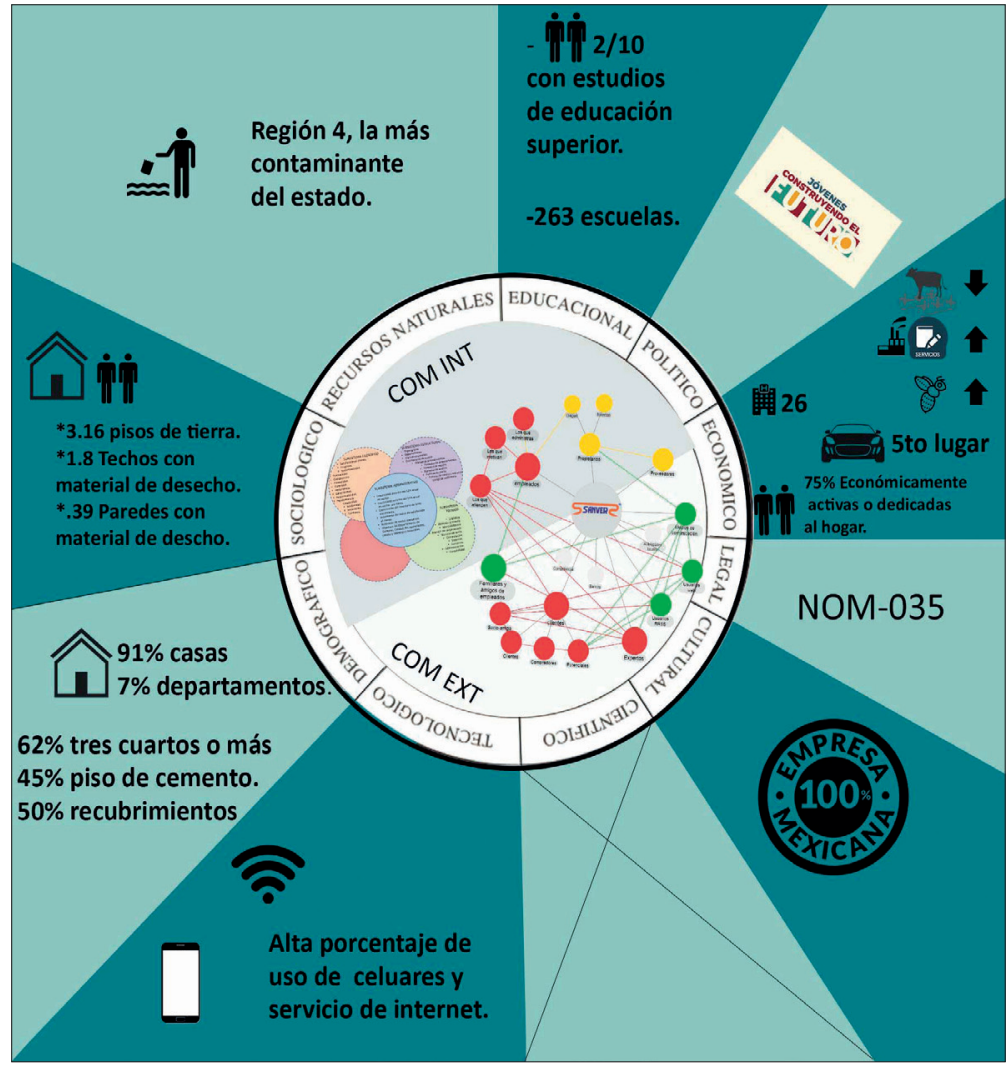

Fuente: elaboración propia / figura adaptada de Scheinsohn (2009), Capriotti (2009), Massoni (2013), Chiesa (2013) \& Kast y Rosenzweig (1990).

Esta primera radiografía deja ver las dimensiones estructurales, funcionales y relacionales de la organización en torno a la FC, que se muestra en la Tabla 1: 
Tabla 1. Lectura del Mapa relacional de la organización Sanver, sucursal Cholula

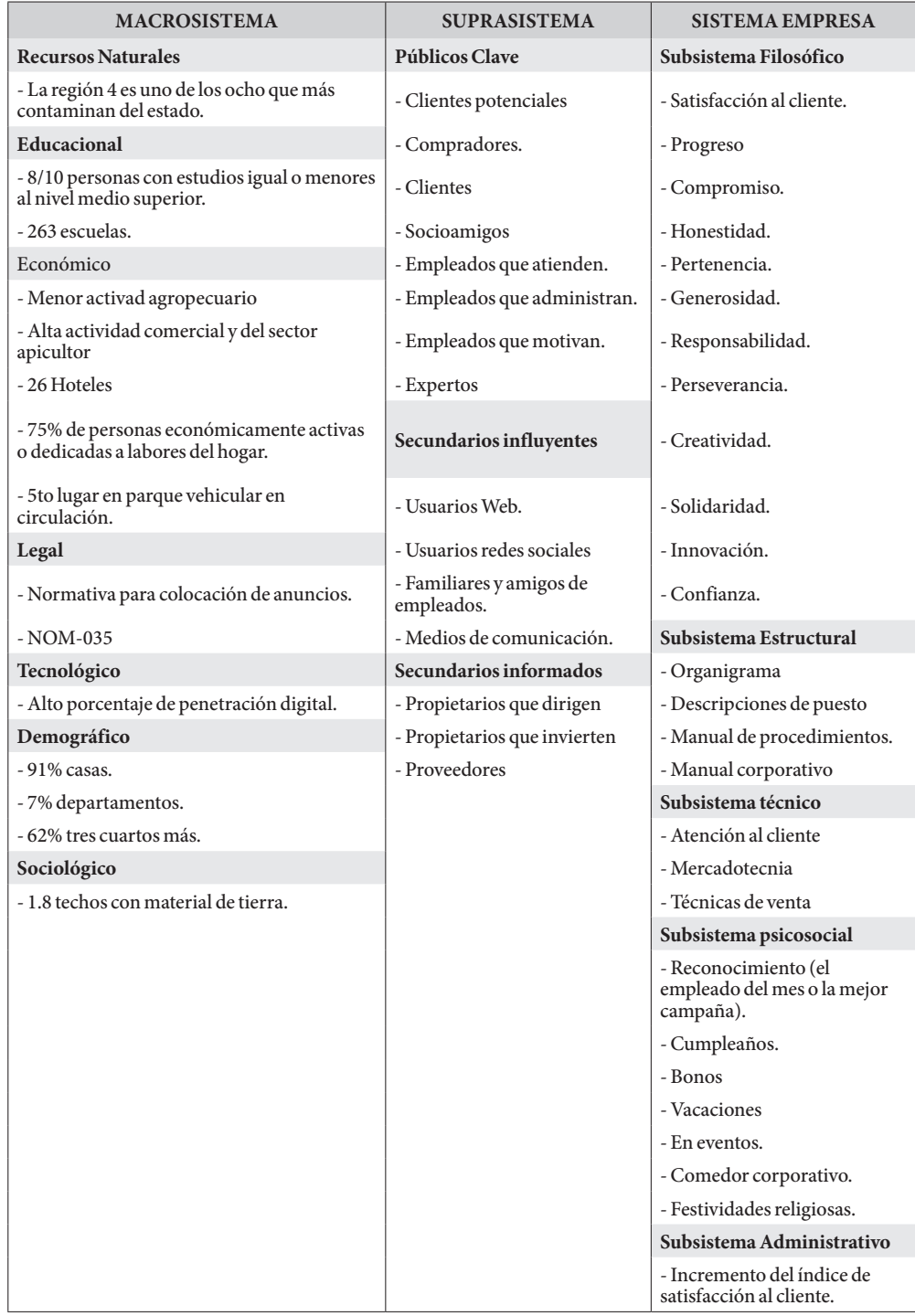

Fuente: elaboración propia.

\subsection{Segundo momento: relaciones de valor compartido}

Para Porter y Kramer (2011), la creación de valor compartido se genera en el momento en el que la empresa logra beneficios tanto organizacionales como sociales, y debe traducirse en competitividad, innovación y responsabilidad so- 
cial. Para lograrlo se requiere de una estrategia corporativa que sea capaz de articular las dimensiones estructurales, funcionales, relacionales y simbólicas de todos los componentes del mapa organizacional, y para ello es necesaria la CC.

En este caso, para identificar las relaciones de valor compartido, que genera una estrategia de comunicación corporativa eficiente y que se traducirá en la fidelización de los clientes, es necesario identificar los elementos que interactúan entre sí. Para ello, el planteamiento se basa en dos perspectivas: el Branding Corporativo de Capriotti (2009) y el Proceso Comercial Relacional de Chiesa (2013). A partir de estas dos miradas se logra establecer las 8 dimensiones que deberán considerarse en el modelo de diagnóstico, expuestas en la Tabla 2:

Tabla 2: Dimensiones de la comunicación corporativa y la fidelización del cliente

\begin{tabular}{|l|l|}
\hline \multicolumn{1}{|c|}{ VARIABLE } & \multicolumn{1}{|c|}{ DIMENSIONES } \\
\hline \multirow{4}{*}{ Comunicación Corporativa } & Identidad \\
\cline { 2 - 2 } & Posicionaiento \\
\cline { 2 - 2 } & Imagen \\
\cline { 2 - 2 } Fidelización del Cliente & Reputación \\
\cline { 2 - 2 } & Atraer \\
\cline { 2 - 2 } & Vender \\
\cline { 2 - 2 } & Satisfacer \\
\cline { 2 - 2 } & Fidelizar \\
\hline
\end{tabular}

Fuente: Elaboración propia, en base a Capriotti (2009) \& Chiesa (2013).

Con base en los planteamientos teóricos, se concluyó que existe una relación dialógica entre la CC y la FC, debido a que ambas se construyen a través de los atributos que los clientes perciben de la organización En ese sentido, Capriotti (2009) plantea que la CC es "el sistema global de comunicación entre una organización y sus diversos públicos" (p. 39). Por lo tanto, la CC es el proceso que une la red de información y de significados de cualquier organización; y que, a su vez, conforma un sistema simbólico que le da orientación y sentido a las acciones e interacciones de la organización en sentido amplio, con todos sus públicos, y en particular con los clientes cautivos.

Al respecto, Ulloa, Apolo y Villalobos (2015) agregan que la CC es el esfuerzo formal que la empresa hace para intercambiar experiencias, conocimientos, emociones y pensamientos entre los sujetos con la finalidad de producir sentidos, crear universos simbólicos y sistemas de significación que contribuyan al logro de objetivos estratégicos. Por ello, la CC representa la parte medular de las relaciones sociales, laborales y comerciales de una organización.

Djenderedjian (2006) señala quela FC depende de "las acciones que permiten conocer en profundidad a los mismos, y a partir de esa información orientar sus percepciones" (p. 2) con la finalidad de forjar relaciones de largo plazo, lo queimplica la creación de interacciones que sólo pueden ser orquestadas desde un sistema formal de CC. 
Mientras que la propuesta teórica de Capriotti (2009) asegura que sólo a través de la gestión de la identidad corporativa es posible incidir en la imagen, en el posicionamiento y en la reputación, debido a que es en la identidad de la empresa donde se puede actuar estratégicamente para lograr la imagen corporativa deseada. Así lo apunta el mismo autor:

Para poder actuar sobrela imagen quetienen los públicos, se hacenecesario desarrollar una adecuada Estrategia de Identidad Corporativa dela organización, que es un proceso metódico y constante de planificación de dicha identidad y dela comunicación dela misma, que permita establecer los parámetros básicos de actuación de la organización. (Capriotti, 2009, p. 132)

Por otra parte, Chiesa (2013) plantea que la FC es el proceso cuyo propósito es establecer lazos estrechos y personalizados que, sobre todo, aporten el valor único necesario para establecer relaciones comerciales sólidas y duraderas.

Con base en estos planteamientos teóricos, se propone un modelo de diagnóstico que logre integrar las variables CC y FC para construir una estrategia más efectiva. En la Figura 3 se muestra el modelo que permite identificar la correlación entre las dimensiones de ambas variables, anclado en el corazón del mapa relacional correspondiente al presente caso de estudio.

Figura 3. Modelo correlacional de la comunicación corporativa y el proceso de fidelización al cliente

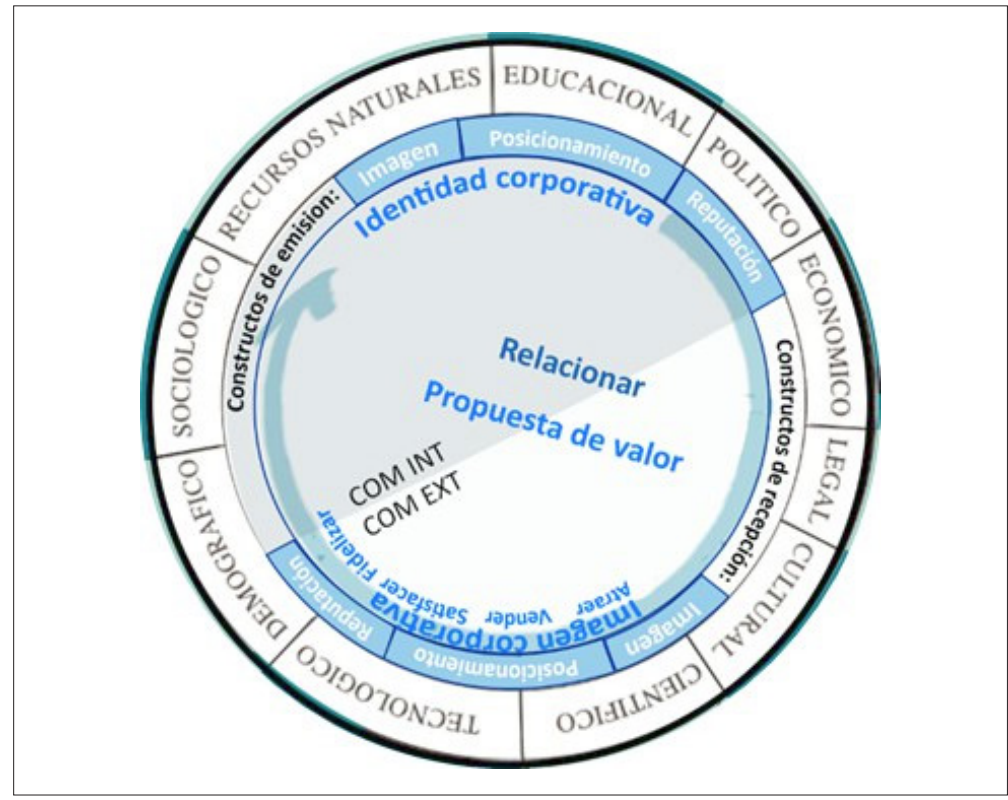

Fuente: Elaboración propia, en base a Capriotti (2009) y Chiesa (2005). 
Estas dimensiones representan el punto de partida para hallar los indicadores y las herramientas que contribuyan al diseño de un modelo estratégico de diagnóstico. El resultado que se busca es identificar la carga informativa, relacional y simbólica que se pone en marcha en el sistema organizacional, para orientarlo a la mejora de la FC de la empresa Sanver, sucursal Cholula.

\section{DISEÑO METODOLÓGICO}

Para Calvo (2006), un modelo es una representación o esquema básico de comportamiento que se construye a partir de un conjunto de enunciados teóricos para sintetizar la información disponible sobre un fenómeno. Para la fundamentación teórica del modelo se analizaron 39 fuentes bibliográficas sobre las dimensiones de cada variable, y tras su análisis se seleccionaron 17 fuentes en las que se hallaron aportaciones que, a su vez, permitieron establecer 14 subdimensiones, con las que fue posible precisar los siguientes objetivos metodológicos e indicadores, clasificados en la Tabla 3:

Tabla 3. Muestra de documentos revisados para integrar la propuesta metodológica del Modelo estratégico para diagnosticar la comunicacional corporativa orientado a mejorar la fidelización del cliente de la empresa Sanver, sucursal Cholula

\begin{tabular}{|c|c|c|c|c|c|}
\hline Variable & Dimensión & $\begin{array}{l}\text { Sub- } \\
\text { dimensión }\end{array}$ & Objetivos metodológicos & Indicadores & Base metodológica \\
\hline \multirow{5}{*}{ 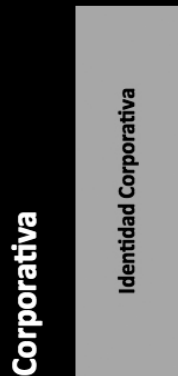 } & \multirow{5}{*}{ 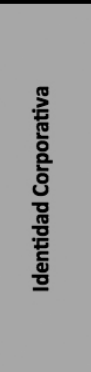 } & $\begin{array}{l}\text { Filosofía } \\
\text { Corporativa }\end{array}$ & \multirow{2}{*}{$\begin{array}{l}\text { Identificar el desfase que existe entre la } \\
\text { Cultura Corporativa deseada y la Cultura y } \\
\text { Clima Coporativa actual }\end{array}$} & \multirow{2}{*}{$\begin{array}{l}\text { Desfase de la cultura } \\
\text { deseada vs Perfil de la } \\
\text { cultura actual }\end{array}$} & \multirow{2}{*}{$\begin{array}{l}\text { Kast \& Rosenzweig } \\
\text { (1990) / Capriotti } \\
\text { (2009) }\end{array}$} \\
\hline & & $\begin{array}{c}\text { Cultura } \\
\text { Corporativa }\end{array}$ & & & \\
\hline & & $\begin{array}{c}\text { Clima } \\
\text { organizacional }\end{array}$ & $\begin{array}{l}\text { 1. Evaluar el nivel de aprovechamiento de } \\
\text { los medios de comunicación interna. } \\
\text { 2. Determinar el tipo de Clima } \\
\text { Organizacional } \\
\text { 3. Conocer los factores que intervienen } \\
\text { positiva y negativamente en el rendimiento } \\
\text { laboral. }\end{array}$ & $\begin{array}{l}\text { Aprovechamiento de } \\
\text { medios de comunicación } \\
\text { interna / Tipo de Clima } \\
\text { Organizacional y } \\
\text { rendimiento laboral. }\end{array}$ & $\begin{array}{l}\text { Márquez (2017) / } \\
\text { ESChool }\end{array}$ \\
\hline & & $\begin{array}{l}\text { Análisis de } \\
\text { públicos }\end{array}$ & $\begin{array}{l}\text { Identificar los públicos claves de la } \\
\text { organización }\end{array}$ & Jerarquización & Capriotti (2009) \\
\hline & & $\begin{array}{l}\text { Análisis del } \\
\text { entorno }\end{array}$ & $\begin{array}{l}\text { Detectar los factores del entorno con mayor } \\
\text { repercusión en la relación con el cliente }\end{array}$ & Factores macrosistémicos & Scheinsohn (2009) \\
\hline$\frac{5}{2}$ & 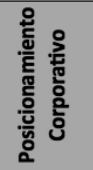 & $\begin{array}{l}\text { Notoriedad } \\
\text { Corporativa }\end{array}$ & $\begin{array}{l}\text { Establacer el grado de conocimiento que } \\
\text { tienen los clientes de la organización }\end{array}$ & $\begin{array}{l}\text { Nivel y Calidad de la } \\
\text { notoriedad. }\end{array}$ & Capriotti (2009) \\
\hline & 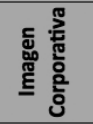 & $\begin{array}{l}\text { Perfil de la } \\
\text { imagen } \\
\text { corporativa }\end{array}$ & $\begin{array}{c}\text { Conocer los atributos que los públicos claves } \\
\text { asocian con el perfil de la organización vs la } \\
\text { competencia }\end{array}$ & Atributos de la Imagen & Capriotti (2009) \\
\hline & 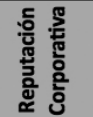 & $\begin{array}{l}\text { Personalidad de } \\
\text { la marca }\end{array}$ & $\begin{array}{l}\text { Conocer los rasgos de personalidad que se } \\
\text { asocia con la marca vs los de la competencia }\end{array}$ & Rasgos de personalidad & $\begin{array}{c}\text { Goñi, Torres \& Aguilera } \\
\text { (2013), basados en } \\
\text { Aeker (1997) }\end{array}$ \\
\hline
\end{tabular}

Fuente: Elaboración propia, en base a: Kast \& Rosenzweig (1990), Capriotti (2009), Márquez (2017), School (2017), Scheinsohn (2009), Goñi, Torres \& Aguilera (2013), Aaeker (2015), Marco \& Visiedo (2012), Massoni (2013), Temkin (2010)companies often neglect customers. But they don't need to. We recommend that organizations use customer journey maps to examine interactions from their customers' points of view. Mapping the customer journey requires five steps: 1, Chiesa (2013), Castillo (2005), Situmorang, Rini \& Muda, (2017). 
Derivados del análisis anterior, se lograron determinar los siguientes resultados:

- La primera variablequedó integrada de 4 dimensiones y 7 subdimensiones, de las que se desprenden 8 objetivos metodológicos, 11 indicadores y 61 escalas, que se examinarán a través de 5 herramientas diseñadas para 3 públicos distintos.

Tabla 4. Tablero de instrumentos metodológicos correspondiente a variable comunicación corporativa

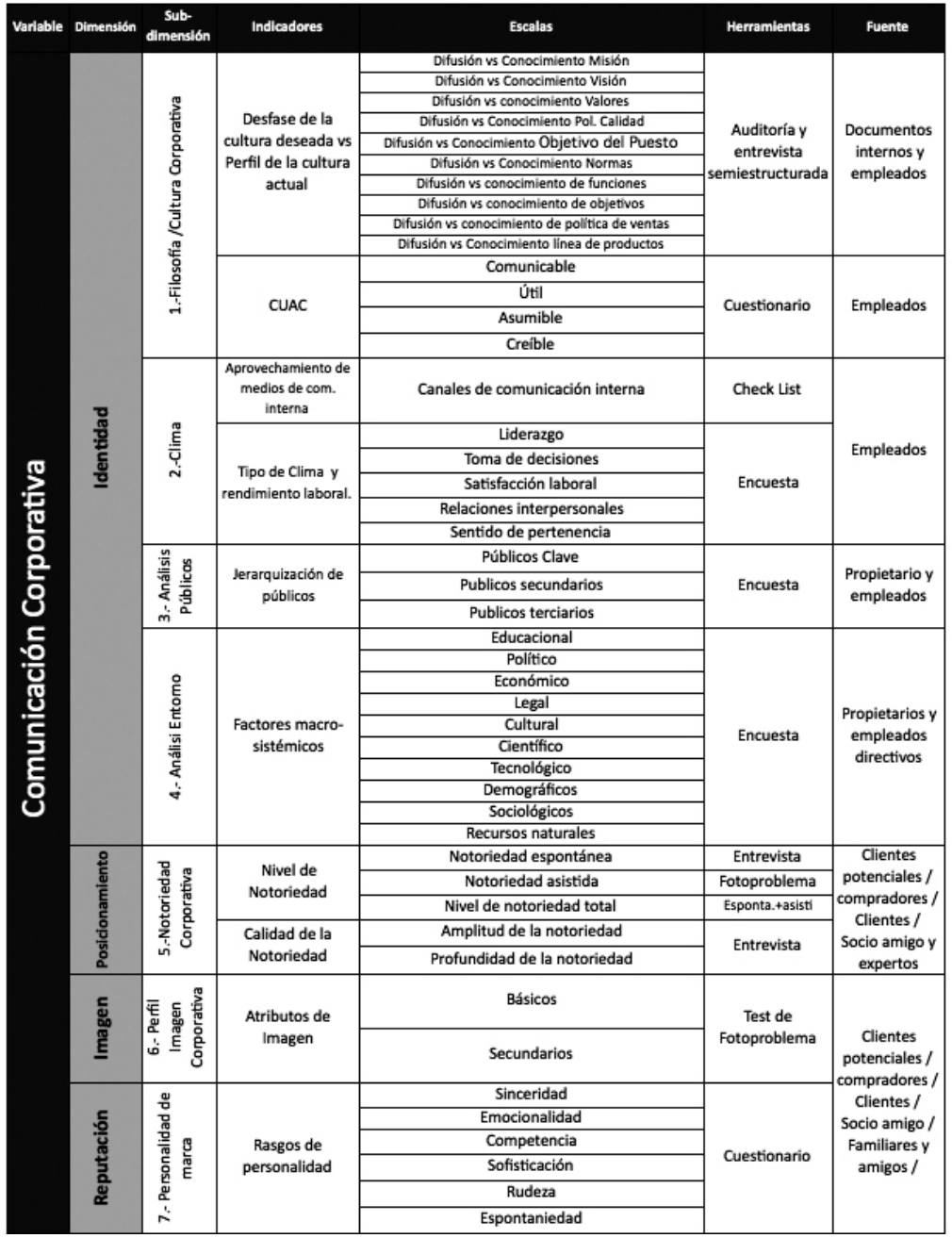

Fuente: Elaboración propia, en base a Kast \& Rosenzweig (1990), Capriotti (2009), Márquez (2017), School (2017), Scheinsohn (2009), Goñi, Torres \& Aguilera (2013), Aaeker (2015). 
La segunda variante arrojó el siguiente resultado:

- Quedó conformada por cuatro dimensiones y seis subdimensiones, de las que se derivan la misma cantidad de objetivos metodológicos e indicadores, 23 escalas y seis diferentes tipos de herramientas para la recolección de datos, aplicadas en su mayoría a los clientes, haciendo la distinción de los cuatro perfiles en los que fueron clasificados: clientes potenciales, compradores, clientes y socioamigos.

Tabla 5. Tablero de instrumentos metodológicos correspondientes a la variable fidelización del cliente

\begin{tabular}{|c|c|c|c|c|c|c|}
\hline Varlable & Dimensión & $\begin{array}{c}\text { Sub- } \\
\text { dimensión }\end{array}$ & Indicadores & Escalas & Herramientas & Fuente \\
\hline \multirow{7}{*}{\multicolumn{2}{|c|}{ 递 }} & \multirow{3}{*}{ 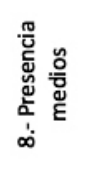 } & $\begin{array}{l}\text { No. de medios } \\
\text { utilizados }\end{array}$ & Lista de medios utilizados & Check List & $\begin{array}{l}\text { Empleados y } \\
\text { propietarios }\end{array}$ \\
\hline & & & $\begin{array}{l}\text { Visibilidad y } \\
\text { Notoriedad }\end{array}$ & Frecuencia mensual & \multirow{2}{*}{ Monitoreo } & \multirow{2}{*}{$\begin{array}{c}\text { Medios de } \\
\text { Comunicación } \\
\text { y RRSS }\end{array}$} \\
\hline & & & Valoración & Valoración & & \\
\hline & & \multirow{4}{*}{ 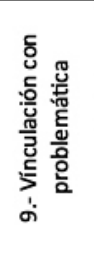 } & Saberes & Conocimiento del problema & \multirow{4}{*}{$\begin{array}{l}\text { Entrevista } \\
\text { semi- } \\
\text { estructurada }\end{array}$} & \multirow{4}{*}{$\begin{array}{c}\text { Clientes } \\
\text { potenciales / } \\
\text { compradores / } \\
\text { Clientes / } \\
\text { Socio amigo y } \\
\text { expertos / } \\
\text { Usuarios de } \\
\text { RRSS y Web }\end{array}$} \\
\hline & & & Intereses & Detectar los intereses & & \\
\hline & & & Necesidades & Conocer necesidades & & \\
\hline & & & Expectativas & Descubrir expectativas & & \\
\hline \multirow{6}{*}{\multicolumn{2}{|c|}{ 这 }} & \multirow{6}{*}{ 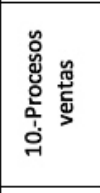 } & \multirow{6}{*}{$\begin{array}{l}\text { Experiencia de } \\
\text { compra }\end{array}$} & Prepara venta & \multirow{6}{*}{ Auditoría } & \multirow{6}{*}{$\begin{array}{c}\text { Ctes } \\
\text { /Socioamnigo }\end{array}$} \\
\hline & & & & Contacto & & \\
\hline & & & & Presentación de la oferta & & \\
\hline & & & & Negociación & & \\
\hline & & & & Cierre / No Cierre & & \\
\hline & & & & Seguimiento de la venta & & \\
\hline \multirow{7}{*}{\multicolumn{2}{|c|}{ 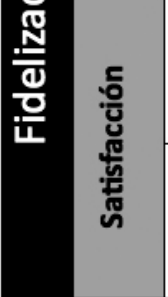 }} & \multirow{3}{*}{ 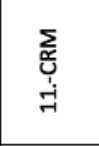 } & \multirow{3}{*}{$\begin{array}{l}\text { Frecuencia de } \\
\text { compra }\end{array}$} & Clientes potenciales & \multirow{3}{*}{$\begin{array}{c}\text { Análisis } \\
\text { estadístico }\end{array}$} & \multirow{3}{*}{$\begin{array}{c}\text { Ctes } \\
\text { /Socioamnigo }\end{array}$} \\
\hline & & & & Compradores & & \\
\hline & & & & $\begin{array}{c}\text { Clientes } \\
\text { Sociamigo }\end{array}$ & & \\
\hline & & \multirow{4}{*}{ 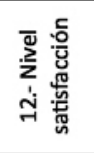 } & \multirow{4}{*}{$\begin{array}{l}\text { Calidad en el } \\
\text { servicio }\end{array}$} & Confiabilidad & \multirow{4}{*}{ Cuestionario } & \multirow{4}{*}{$\begin{array}{c}\text { Ctes } \\
\text { /Socioamnigo }\end{array}$} \\
\hline & & & & Responsabilidad & & \\
\hline & & & & Seguridad & & \\
\hline & & & & $\begin{array}{c}\text { Empatía } \\
\text { Bienes materiales }\end{array}$ & & \\
\hline & 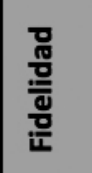 & & $\begin{array}{c}\text { Identifiación/ } \\
\text { INetpromotescore }\end{array}$ & Nivel de recomendación & Cuestionario & $\begin{array}{c}\text { Clientes / } \\
\text { socioamigo / } \\
\text { Familiares y } \\
\text { amigos }\end{array}$ \\
\hline
\end{tabular}

Fuente: Elaboración propia, en base a referencias de Marco \& Visiedo (2012), Massoni (2013), Temkin (2010)companies often neglect customers. But they don't need to. We recommend that organizations use customer journey maps to examine interactions from their customers' points of view. Mapping the customer journey requires five steps: 1, Chiesa (2013), Morales (2005), Situmorang, Rini \& Muda, (2017). 
El Tablero de instrumentos metodológicos es un compendio que ofrece una guía metodológica y que, al vincularse con el apartado teórico, integra la propuesta del Modelo Estratégico de Diagnóstico de la CC para mejorar la FC de la empresa Sanver, sucursal Cholula, tal como se observa en la Figura 4.

Figura 4. Modelo estratégico de diagnóstico de la comunicación corporativa orientado a mejorar la fidelización del cliente de la empresa Sanver, sucursal Cholula

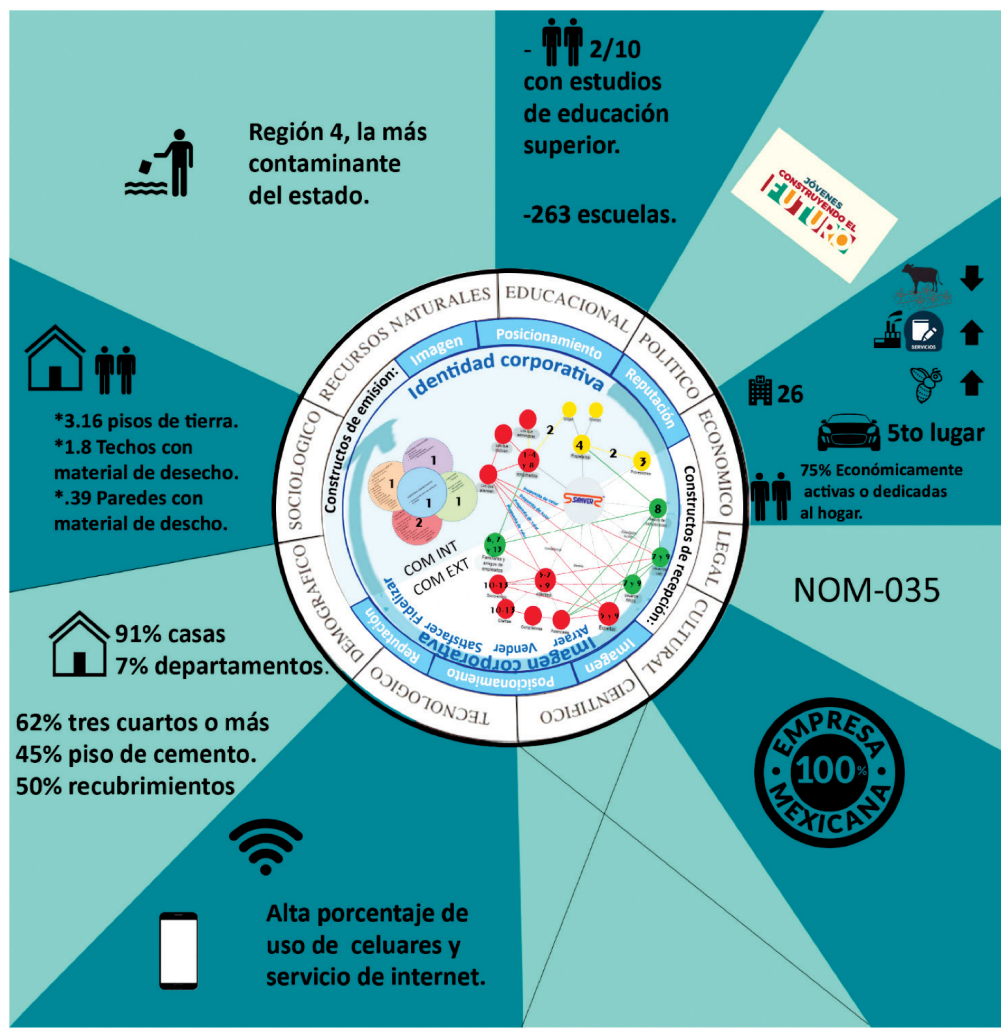

Fuente: elaboración propia.

\section{CONCLUSIONES}

El Modelo estratégico de diagnóstico de la CC para la FC se apoya en dos ejes fundamentales: la visión multidimensional de la empresa, representada en el mapa estratégico organizacional, y la noción de valor en la construcción de relaciones sólidas, responsables y sostenibles. Para ello se requieren diversos cambios en los paradigmas dominantes de la gestión comunicacional de las organizaciones. 
Por una parte, es necesario partir de una perspectiva teórica de la CC que abandone la tradición informacional y se acerque a la visión relacional del proceso comunicacional. Por otra, es necesario una conceptualización de la FC que trascienda el momento de la venta y a la construcción de vínculos de valor; y finalmente, derivado de esos cambios, también es necesario un esfuerzo metodológico complejo, con un paquete diverso de técnicas e instrumentos que logre recabarla información suficiente para orientar la gestión comunicacional.

Con base en los planteamientos teóricos que se describieron a lo largo del artículo, se concluyó que existe una relación dialógica entre la CC y la FC dado que ambas se construyen a través de los atributos y los valores que los clientes perciben de la organización. El Modelo estratégico de diagnóstico logra integrar estas perspectivas, abandonando la idea reduccionista que considera a la CC como una serie de actividades informativas y lineales, para concebirla como un sistema funcional que construye una red de experiencias y significados, capaz de producir un ambiente simbólico, que contribuye al logro de los objetivos de fidelización. Además, conceptualiza la FC como un proceso que pretende forjar vínculos comerciales sólidos, duraderos y que aporten valor al cliente, a la organización y al entorno social. Esto se logra en el momento en quela CC orienta estratégicamentelos activos intangibles-comola identidad, la imagen yla reputación dela organización-hacialos programas deatracción, satisfacción y fidelización.

En este sentido, es necesario considerar el sistema de CC como un proceso que garantiza la articulación continua de la carga informativa, relacional y simbólica, a fin de convertir a la organización en una CC que traduzca sus relaciones de valor compartido en competitividad, innovación y responsabilidad social.

\section{REFERENCIAS}

Aaeker, J. (2015). Dimension of bran personality. Cosmos. DOI: https://doi. org/10.2307/3151897

Apolo, D., Báez, V., Pauker, L. \& Pasquel, G. (2017). Gestión de Comunicación Corporativa: consideraciones para el abordaje de su estudio y práctica. Revista Latina de Comunicación Social, (72), 521-539. DOI: https://doi.org/10.4185/RLCS

Calvo,D.(2006).Modelosteóricosyrepresentacióndelconocimiento. Madrid:Universidad Complutense de Madrid.

Capriotti, P. (2009). Branding Corporativo: fundamentos para la gestión estratégica de la identidad corporativa. Madrid: Andros.

Castelló-Martínez, A.(2010). La orientación empresarial hacia el cliente en la Web 2.0. MHCJ, 1, 99-121. 
Castillo, E. (2005). Escala multidimensional SERVQUAL. Recuperado de: https://www. gestiopolis.com/wp-content/uploads/2010/03/mida-la-calidad-de-su-serviciocon-la-escala-servqual.pdf

Chiesa, C. (2005).CRM las cincopirámides del marketing relacional: Cómoatraer, vender, satisfacer y fidelizar clientes de forma rentable. España: Ed. Deusto.

Chiesa, C. (2013). Vender es mucho más. Madrid: Empresa Activa.

Djenderedjian, J. C. (2006). Estrategias de Captación y Fidelización de Clientes en un Medio Competitivo. Crédito, Moneda y Comercio Rural en el Sur Entrerriano a Fines de la Colonia. Anuario IEHS, 21, 287-310.

Garrido, J. (2007). Las claves de la comunicación empresarial en el siglo XXI. Madrid: Ediciones Gestión 2000.

Goñi, N., Torres, E. \& Aguilera, S. (2013). Dimensiones de la personalidad de la marca en México. Revista de Ciencias Sociales (RCS), XIX(2), 213-225.

Kast, F. E. \& Rosenzweig, J. E. (1990). Enfoque de Sistemas y de Contingencias. México: MC Graw-Hill.

Marco Crespo, E. (2011). La dimensión mediática de la reputación corporativa, su gestión y evaluación: diez años de la Universidad CEU Cardenal Herrera en la prensa valenciana. Tesis doctoral dirigida por la Dra. Rosa Visiedo Claverol, Departamento de Comunicación e Información Periodística, Universidad Cardenal Herrera-CEU, Valencia, España.

Marketing,P. (2018).Lafidelización, clavepara aumentarla rentabilidad delasempresas. Recuperado de: https://inloyalty.es/publicaciones/2019/06/11/atraccion-oretencion-conoce-mas-sobre-el-primer-barometro-de-fidelizacion

Márquez, D. C. (2017). Estudio de clima organizacional en la Cooperativa de ahorro y crédito CACPE Zamora Ltda. Tesis para el título de Psicología Organizacional dirigida por el Mg. Mario Moyano Moyano, Facultad de Filosofía, Letras y Ciencias de la Educación, Universidad del Azuay, Cuenca, Ecuador.

Massoni, S. (2013). Metodologías de la comunicación estratégica. Del inventario al encuentro sociocultural. Rosario: Homo Sapiens.

Porter, M. E. \& Kramer, M. R. (2011). The Big Idea: Creating Shared Value. How to reinvent capitalism - and unleash a wave of innovation and growth. Harvard Business Review, 89(1-2), 62-77.

Scheinsohn, D. (2009). Comunicación Estratégica I. Buenos Aires: Granica SA.

School, E. B. (2017). Medios y Canales para la comunicación interna corporativa. Recuperadode:https://www.eae.es/actualidad/noticias/medios-y-canales-parala-comunicacion-interna-corporativa 
Situmorang,S.H., Rini,E.S. \&Muda,I. (2017).Customerexperience, netemotionalvalue and net promoter score on muslim middle class women in medan. International Journal of Economic Research, 14(20), 269-283.

Temkin, B. D. (2010). Mapping The Customer Journey Best Practices For Using An Important Customer Experience Tool. Forrester, 20. Available on: http:// crowdsynergy.wdfiles.com/local--files/customer-journey-mapping/mapping_ customer_journey.pdf

Ulloa, C., Apolo, D. \& Villalobos, J. (2015). Aproximación conceptual a la comunicación corporativa: retos y propuestas. Austral Comunicación, 4(2), 287-301. DOI: https://doi.org/10.26422/137

\section{OTRAS REFERENCIAS}

Dir\&Age. (2018). El reto de retenery fidelizar clientes en la pyme. Recuperado de: https:// directivosygerentes.es/pymes/noticias-pymes/fidelizar-clientes-pyme

* Contribución: El trabajo fue organizado de manera conjunta y se distribuye en porcentajes similares.

* Nota: El editor Paul Capriotti y el Comité Editorial de la revista aprobaron la publicación del artículo.

\section{(cc) BY}

Artículo publicado en acceso abierto bajo la Licencia Creative Commons - Attribution 4.0 International (CC BY 4.0). 


\section{IDENTIFICACIÓN DE LES AUTORES}

Patricia Durán Bravo. Doctora en Comunicación Aplicada por la Universidad Anáhuac del Norte, México. Profesionalmente ha colaborado en medios de comunicación, agencias de publicidad y empresas de sector de la transformación coordinando las funciones de comunicación interna relaciones públicas y capacitación. Fue docente investigadora de la Benemérita Universidad Autónoma de Puebla (BUAP), México. Es Miembro de la Red Mexicana de Investigadores en Estudios Organizacionales (REMINEO), la Asociación Iberoamericana de Sociología de las Organizaciones y Comunicación (AISOC) y la Red Internacional de Investigación y Consultoría en Comunicación (RIICC). Actualmente, combina su carrera docente con la de consultora en diversas instituciones de carácter privado, gubernamental y social. Es coautora de diversos libros especializados y es ponente en eventos a nivel nacional e internacional en el campo de la Comunicación Estratégica. Entre sus trabajos se pueden mencionar: La comunicación interna en la implementación del Modelo Universitario Minerva (MUM) de la BUAP, 2009. Una propuesta de análisis -coautoría con A. Mendieta Ramírez- (2009); Comunicación Estratégica: construcción comunicacional de la estrategia corporativa en organizaciones sociales -coautoría con N. G. Cisneros Martínez, V. M. Meléndez Rodríguez y M. Á. Leonor Martínez- (2016).

Nancy Cisneros Martínez. Doctora en Imagen, Arte, Cultura y Sociedad por la Facultad de Diseño de la Universidad Autónoma de Morelos, México. Docente de la Maestría en Comunicación Estratégica de la Benemérita Universidad Autónoma de Puebla (BUAP), México. Miembro del Cuerpo Académico Comunicación en la Organizaciones de la Facultad de Ciencias de la Comunicación de la BUAP. Sus líneas de investigación son: comunicación estratégica y comunicación alternativa. Es Miembro de la Red Mexicana de Investigadores en Estudios Organizacionales (REMINEO), A. C., y del Colegio de Investigadores. Miembro de la Asociación Iberoamericana de Sociología de las Organizaciones y Comunicación. Miembro de la Red Internacional de Investigación y Consultoría en Comunicación (RIICC). Ponente a nivel nacional e internacional. Autora y coautora de diversos artículos, entre ellos: Comunicación estratégica: articulación de las ecologías simbólicas con los stakeholders -coautoría con R. Pancardo Peralta y P. Durán Bravo- (2019, Fondo de Publicaciones Universidad Sergio Arboleda).

Raúl Pancardo Peralta. Estudiante dela Maestría en Comunicación Estratégica dela Benemérita Universidad Autónoma de Puebla (BUAP), México. Experiencia laboral enla coordinación de generación de contenidos digitales para empresas, medios, dependencias de gobierno y funcionarios públicos. Ha sido responsable del área de mercadotecnia y de atención a clientes para una empresa dedicada a la distribución de productos por mayoreo a nivel nacional y de venta al retail en México.

\section{REGISTRO BIBLIOGRÁFICO}

Durán Bravo, P., Cisneros Martínez, N. \& Pancardo Peralta, R. (enero-junio, 2020). Comunicación corporativa para la fidelización del cliente. InMediaciones de la Comunicación, 15(1), 183-200. 